\title{
A Neuron Model Based Ultralow Current Sensor System for Bioapplications
}

\author{
A. K. M. Arifuzzman, Mohammad Shafquatul Islam, and Mohammad Rafiqul Haider \\ Department of Electrical and Computer Engineering, The University of Alabama at Birmingham, Birmingham, AL 35294, USA \\ Correspondence should be addressed to Mohammad Rafiqul Haider; mrhaider@uab.edu
}

Received 30 July 2015; Revised 26 January 2016; Accepted 27 January 2016

Academic Editor: Jian-Nong Cao

Copyright ( 2016 A. K. M. Arifuzzman et al. This is an open access article distributed under the Creative Commons Attribution License, which permits unrestricted use, distribution, and reproduction in any medium, provided the original work is properly cited.

An ultralow current sensor system based on the Izhikevich neuron model is presented in this paper. The Izhikevich neuron model has been used for its superior computational efficiency and greater biological plausibility over other well-known neuron spiking models. Of the many biological neuron spiking features, regular spiking, chattering, and neostriatal spiny projection spiking have been reproduced by adjusting the parameters associated with the model at hand. This paper also presents a modified interpretation of the regular spiking feature in which the firing pattern is similar to that of the regular spiking but with improved dynamic range offering. The sensor current ranges between $2 \mathrm{pA}$ and $8 \mathrm{nA}$ and exhibits linearity in the range of 0.9665 to 0.9989 for different spiking features. The efficacy of the sensor system in detecting low amount of current along with its high linearity attribute makes it very suitable for biomedical applications.

\section{Introduction}

In light of the successful strides achieved in biomedical technology in the last couple of decades, the 21st century is experiencing intensified demands in ultralow current biosensors as it has become increasingly apparent that ultralow current sensors play a critical role in many bioapplications, especially those aimed at biosensing systems. The ultralow current sensors are frequently used in areas such as clinical diagnosis, genome research, drug development [1], surveying systems, metabolite activity monitoring [2], and bioelectrochemical sensors [3].

State-of-the-art biosensors offer numerous advantages in the form of high selectivity, high sensitivity, large dynamic range, lower response time, simple calibration techniques, field reconfigurability, reproducibility, stability, low power consumption, and low manufacturability cost. A neuron's capability of sensing changes in environment is exceptionally explicit and exquisitely sensitive [4], doing such with flying initial response times, which are usually as short as milliseconds. A lot of effort has already been put into the advancement of spike based neuron sensor applications such as tactile sensing [5], biomolecular detections [6], and capacitive biosensor [7]. Due to the compensations mentioned above an ultralow current detection sensor based on neural spiking model has been introduced in this paper.

The neuron spiking model is based on the Izhikevich neuron model which offers similar biological plausibility as the Hodgkin-Huxley model but with superior computational efficiency as the integrate-and-fire model. The mathematical model of the Izhikevich neuron model is given in [8]. A crucial advantage of this model is that one can easily generate different spiking features of a neuron by varying only a handful number of parameters, as we have produced four different spiking features: regular spiking, chattering, neostriatal spiny projection, and modified regular spiking. Among them the modified regular spiking is analogous to the regular spiking feature but with improved range of input sensing current. A notable observation here is that the spiking features of this particular neuron model are always triggered at ultralow current, usually in the picoampere (pA) to nanoampere (nA) 


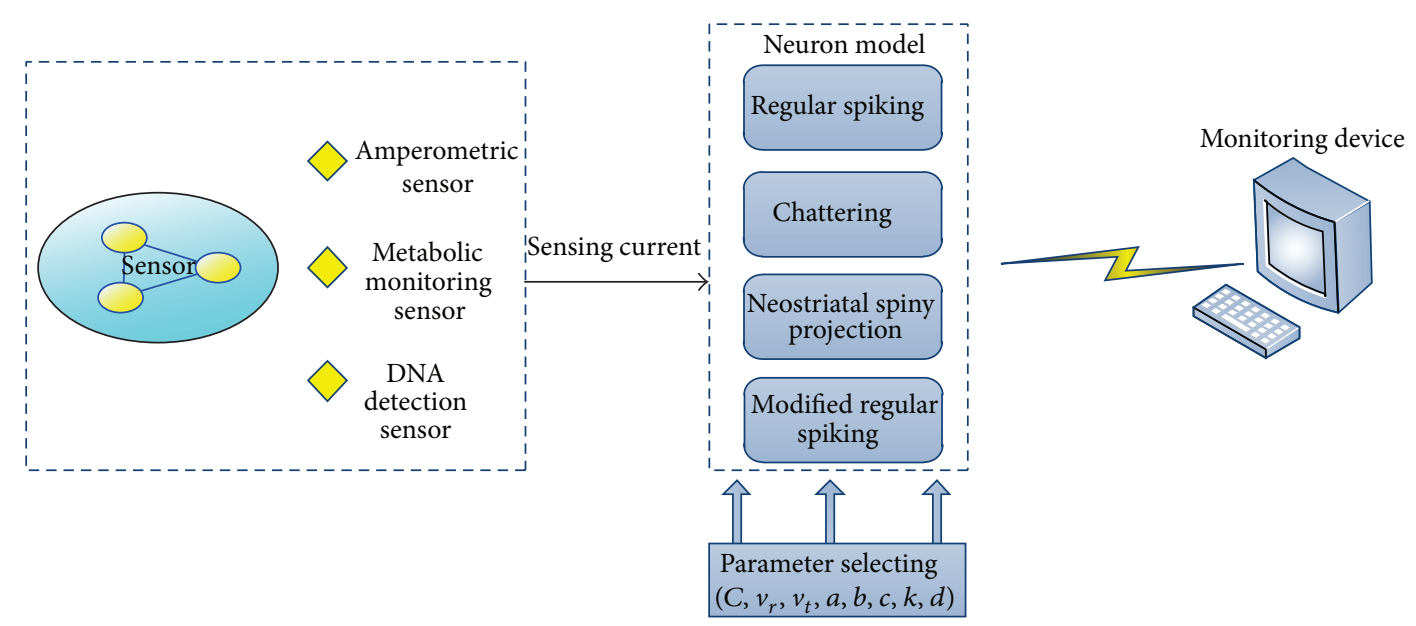

FIGURE 1: System overview of the proposed neuron model based low current sensing system.

range, rendering it a very suitable candidate for ultralow current sensing applications. Hence, in this paper a neural spiking based biosensor has been proposed which has a $2 \mathrm{pA}$ to $8 \mathrm{nA}$ input sensing current range with high linearity.

This paper is organized as follows. Sections 2 and 3 briefly describe the model architecture and sensor characteristics, respectively, while Section 4 introduces the Izhikevich neuron model and explains the four spiking features mentioned above. Section 5 illustrates the results of the neuron model output frequency spectrum and the linearity of the four extracted spike features. Finally, in Section 6, the paper is summarized.

\section{Sensor System Architecture}

Due to the progressive strides made in biomedical technology, especially in the field of biosensor research and production, the significance and demand for innovative and efficient ultralow current sensors are more than ever before. The overall low current sensor architecture of the proposed system model consists of the three blocks, sensor, neuron model, and monitoring device, which has been presented in Figure 1. Bioelectrochemical sensors are widely used to determine analyte concentrations as such measurements are crucial for the study of biological systems along with the development of tools used in disease diagnosis and treatment. On the other hand, with the help of amperometric sensors, it is possible to measure oxygen concentration in blood. There are other biosensors that include DNA sensors and metabolite monitoring sensors. The DNA microarrays developed in recent years are comprehensively used in the field of genome research and drug development. Moreover, remote monitoring systems can be employed to monitor various metabolites in an animal model [2]. The current detected by sensor is fed to a neuron model which in turn fires different spike features, where the frequency of the spikes can be controlled by this firing input current. Neocortical neurons in the mammalian brain can be classified into several types of prominent features according to the pattern of spiking and bursting observed in intracellular recordings. Among the various features of biological spiking neuron, the following neuron spiking patterns are extracted: regular spiking, chattering, and neostriatal spiny projection. The Izhikevich neuron model has been used for its superior computational efficiency and greater biological plausibility. All the spiking features mentioned above have been generated by modifying the parameters associated with the model at hand. This paper introduces a modified version of the regular spiking feature achieved by altering the model parameters. The modified regular spiking firing pattern is similar to that of the regular spiking but with upgraded dynamic range. Lastly the output of the mentioned spiking features is simply a variation in frequency permitted by the input sensing current which is examined in a monitoring device.

\section{Sensors}

It has become increasingly apparent that ultralow current sensors play a critical role in many bioapplications, especially those aimed at biosensing systems. Due to the biomedical technology advances since the past century, ultralow current sensor is believed to be one of the most promising industries in the coming decades along with micro- and nanoelectronics industries. Such developments in biosensor research and production have greatly aggravated the demand of ultralow current sensors which are frequently used in areas such as clinical diagnosis, DNA probing, surveying systems, and protein activity monitoring. In recent times, electrochemical biosensors are being extensively employed to determine analyte concentrations in both research and commercial applications [9], as analyte concentration measurements are essential for the study of biological systems and the development of tools for disease diagnosis and 
treatment. These bioelectrochemical sensors have the ability to identify the protein activity both directly [10] and indirectly through reaction coupling $[11,12]$ to greatly enhance the range of protein classes that can be used as biological recognition elements (BREs). Moreover, an amperometric chemical sensor can be used to measure the concentration of oxygen in blood [13]. In addition to the amperometric chemical sensors, there are other biosensors that are being reported by many distinguished literatures such as metabolite monitoring sensors [2] and DNA molecule based biosensors $[14,15]$.

3.1. Amperometric Sensors. Although optical measurement techniques are widely used in biosensor technology, BREs with electrically coupled outputs offer quite a few benefits which include less or no sample preparation requirement and elimination of performance limiting optical interference and its suitability for chip-level integration of the whole analysis platform [3]. However, to enable cost effective implantation of such high performance bioelectrochemical sensor arrays, some breakthroughs have to be achieved first. In order to accommodate the variable response of various BREs, the system interface must support a large signal range. A bioelectrochemical circuit array microsystem usually consists of a reference electrode, a counter electrode, and an array of working electrodes. The amperometric readout system and the electrode drive system work in tandem to realize the electrochemical potentiostat functions. The electrodes have to be compatible with post-CMOS processing as they must also sustain a reliable interface with subsequently applied biological materials. For the purpose of amperometric detection, most bioelectronics interfaces require an input current ranging from $10 \mathrm{pA}$ to $10 \mu \mathrm{A}$ [3].

3.2. Metabolite Monitoring Sensors. As the name suggests, a metabolite monitoring sensor is capable of detecting single-metabolites in an animal model. Remote metabolism monitoring systems can monitor different metabolites such as glucose, lactate, glutamate, and Adenosine Triphosphate (ATP) [2]. Lately, ATP has been linked with the regulation of adaptive immune responses [16] while glutamate is generally associated with brain damage [17], which itself is a crucial neurotransmitter. To improve the performance of metabolite monitoring sensors, different nanomaterials have been considered such as graphene [18], nanoparticles [19], conductive polymers [20], and Carbon Nanotubes (CNT) [21]. CNT, of late, has demonstrated its ability to enhance the sensitivity for exogenous [22] and endogenous [23] metabolites. The bionanosensor in [2] consists of two coimmobilized enzymes such as glucose oxidase and hexokinase, to monitor the ATP levels. This sensor demonstrates an ATP sensitivity of $34 \mathrm{pA} / \mu \mathrm{M} \mathrm{mm}^{2}$ and a range of $200 \mu \mathrm{M}$ to $1400 \mu \mathrm{M}$, which is in sync with our proposed sensor model.

3.3. DNA Sensors. In recent biotechnology development, DNA sensor has been more important in genetic research such as cancer or hereditary disease, medicine development, detection of infectious agents, and identification in forensic cases [24-27]. DNA sensor array investigates the absence or presence of specific DNA sequence in given sample DNA. DNA microsensor chip is usually made of polymer material, glass, silicon, and so forth. Nowadays, most DNA sensor uses polymerase chain reaction (PCR) amplification but it has some limitations like being expensive, complex, time consuming, and so forth. To overcome those limitations, a new technique has been developed which is biobarcode DNA sensor [28]. The biobarcode DNA sensor system is used in both protein and nucleic acid detection. The DNA microsensor detected current ranges from $10^{-12} \mathrm{~A}$ to $10^{-7} \mathrm{~A}$, which is in agreement with the proposed sensor model.

\section{Neuron Model and Architecture}

In recent times one can observe a swing of emphasis in the artificial neural network community in the direction of spiking neural networks. Inspired by biological innovations, pulse-coupled neural networks alongside spike-timing are considered by numerous studies as a crucial component in the information processing performed by the brain. To get a proper understanding of how the brain actually works, it is essential that the experimental studies of animal and human nervous system are incorporated with numerical simulation of large scale brain models. Twenty of the most prominent features of biological spiking neurons, such as tonic spiking, regular spiking, phasic spiking, chattering, tonic bursting, phasic bursting, spike frequency adaptation, spike latency, and subthreshold oscillations, have been reviewed in [29], which illustrated the richness and complexity of the spiking behavior of individual neuron in response to simple dc current pulses. Before deciding on putting a particular neural model to use, it has to testify that the model is biophysically meaningful with measurable parameters while at the same time exhibiting autonomous chaotic activity. In [29], a comparison of the neurocomputational properties has been presented of several well-known spiking neuron models consisting of integrate-and-fire, integrate-and-fireor-burst, resonate-and-fire, Izhikevich, Morris-Lecar, Wilson, Hodgkin-Huxley, and so forth. It is evident from the comparison that the Hodgkin-Huxley model [30] is the most biophysically accurate but computationally prohibitive due to the fact that it can simulate a very limited number of neurons in real time. While the integrate-and-fire model is computationally effective, it is unrealistically simple and fails to reproduce most of the neurocomputational features exhibited by cortical neurons. The motivation for selecting the Izhikevich neuron model is that it offers the same level of biological plausibility as the Hodgkin-Huxley model while being computationally efficient as the integrate-and-fire model. The Izhikevich model is simple enough in a computational perspective and capable of replicating the rich firing patterns exhibited by real biological neurons. Bifurcation methodologies [8] offer the ability to condense the various biophysically accurate Hodgkin-Huxley type neuron models 


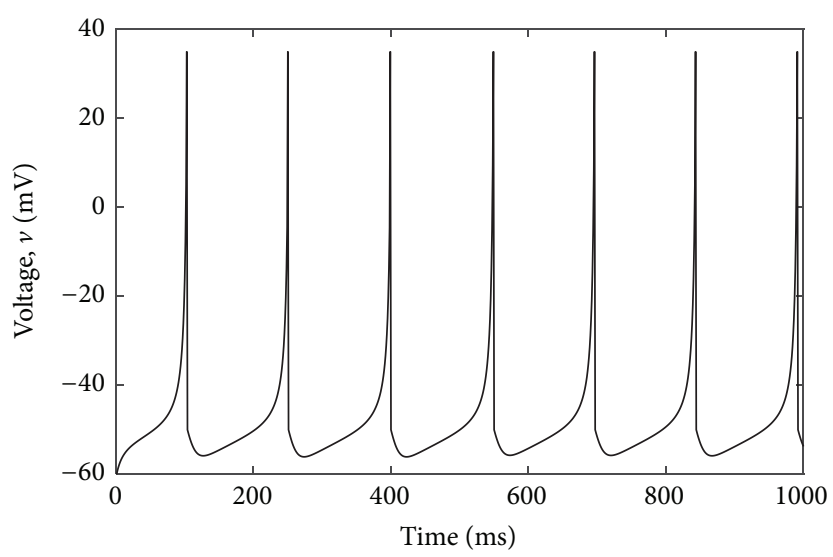

FIGURE 2: Spiking pattern of regular spiking neuron at $70 \mathrm{pA}$.

into a $2 \mathrm{D}$ system of ordinary differential equations which is given in the following:

$$
\begin{aligned}
C \frac{d v}{d t} & =k\left(v-v_{r}\right)\left(v-v_{t}\right)-u+I, \\
\frac{d u}{d t} & =a\left\{b\left(v-v_{r}\right)-u\right\},
\end{aligned}
$$

with the after-spike resetting

$$
\begin{aligned}
& \text { if, } v>v_{\text {peak }}, \quad \text { then, } v \longleftarrow c, \\
& u \longleftarrow u+d,
\end{aligned}
$$

where $v$ and $u$ are dimensionless variables that represent a neuron's membrane potential and membrane recovery current, respectively. The latter is accounted for the activation of $\mathrm{K}^{+}$ionic currents and inactivation of $\mathrm{Na}^{+}$ionic currents, while also providing negative feedback to $v$. According to (4), both $v$ and $u$ are reset after the spike reaches its peak. Constant $k$ can be found when a neuron's rheobase and input resistance are known. $v_{\text {peak }}$ is the voltage that aids us in avoiding amplitude jitter related to the finite time step in the simulations. In the equation above, $C, v_{r}$, and $v_{t}$ are the membrane capacitance, the resting membrane potential, and the instantaneous threshold potential, respectively. $I$ is the synaptic or injected current from a sensor. Equation (1) enables us to produce a waveform in which all the spike feature information is available and from which it is possible to find the frequency of the spikes. The frequency of the spikes can be altered by the sensor current, $I$. Last but not least $a, b$, $c$, and $d$ are parameters which are dimensionless in nature. Parameter $a$ describes the time scale of the recovery variable $u$. Smaller values of $a$ will in fact result in slower recovery. Parameter $b$ represents the sensitivity of the recovery variable $u$ to the subthreshold fluctuations of the membrane potential $v$. Higher values of $b$ will couple $v$ and $u$ more strongly which in turn will result in possible subthreshold oscillations and low-threshold spiking dynamics. The sign of this parameter, whether positive or negative, determines if $u$ is an amplifying variable or not. Parameter $c$ signifies the after-spike reset value of $v$ resulting from the high threshold $\mathrm{K}^{+}$conductances. Parameter $d$ is designated to the after-spike reset of the recovery variable $u$. All these parameters can be easily tailored to replicate any particular neuron spiking feature. In this study, such parameters have been varied to simulate the following neural firing patterns: regular spiking, chattering, neostriatal spiny neuron, and modified regular spiking.

4.1. Regular Spiking. Regular spiking neurons fire tonic spikes that adapt their frequency in response to the sensor current. Morphologically, these particular neurons are spiny stellate cells found in layer 4 and pyramidal cells from layers 2, 3, 5, and 6 [8]. To extract the qualitative and quantitative features of typical regular spiking neurons, the resting membrane potential of $v_{r}=-60 \mathrm{mV}$ and the instantaneous threshold potential of $v_{t}=-40 \mathrm{mV}$ have been perceived. Any instantaneous value above $-40 \mathrm{mV}$ causes the neuron to fire. The rheobase and the input resistance have been set at $50 \mathrm{pA}$ and $80 \mathrm{M} \Omega$, respectively, which result in $k=0.7$ and $b=-2$. The membrane capacitance, $C=100 \mathrm{pF}$, yields a membrane time constant value of $8 \mathrm{~ms}$. The value of $d$ is 100 . Due to the negative value of $b$, depolarization of $v$ decreases the value of $u$. The inactivation time constant, $I_{A}$, is approximately $30 \mathrm{~ms}$ in the subthreshold voltage range; hence $a=0.03 \sim 1 / 30$. During a spike the membrane potential, $v$, of a typical regular spiking neuron reaches a maximum or peak value of $v_{\text {peak }}=$ $+35 \mathrm{mV}$ which is shown in Figure 2. The voltage reset value, $c$, is set at $-50 \mathrm{mV}$ and the input current is $70 \mathrm{pA}$.

4.2. Chattering. Chattering neurons are commonly referred to as Fast Rhythmic Bursting (FRB) due to their ability to fire high frequency bursts of spikes which have relatively short interburst periods. The magnitude of the sensor current determines the interburst period. So it is safe to say that one can control the interburst period by leveraging the amount of current that flows through the sensor in use. Such neurons are found in the visual cortex of adult cats and morphologically they are spiny stellate or pyramidal neurons of layers 2, 3, 


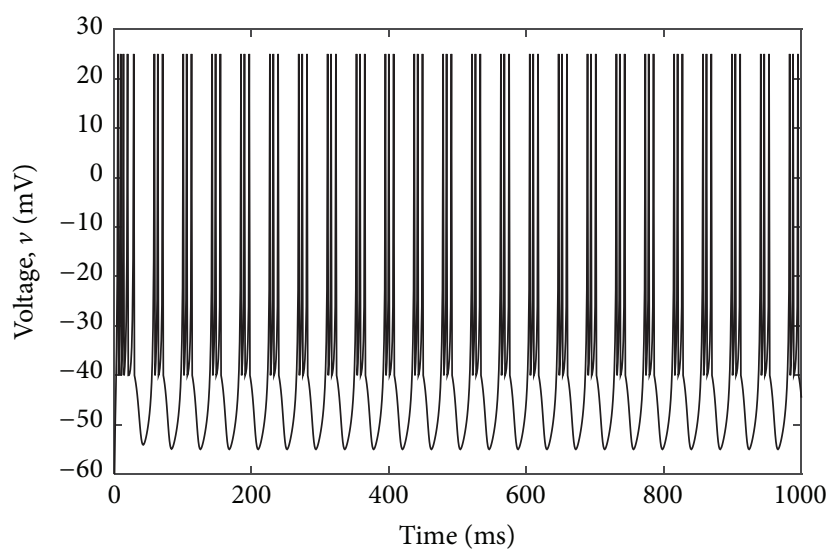

FIGURE 3: Spiking pattern of chattering neuron at $500 \mathrm{pA}$.

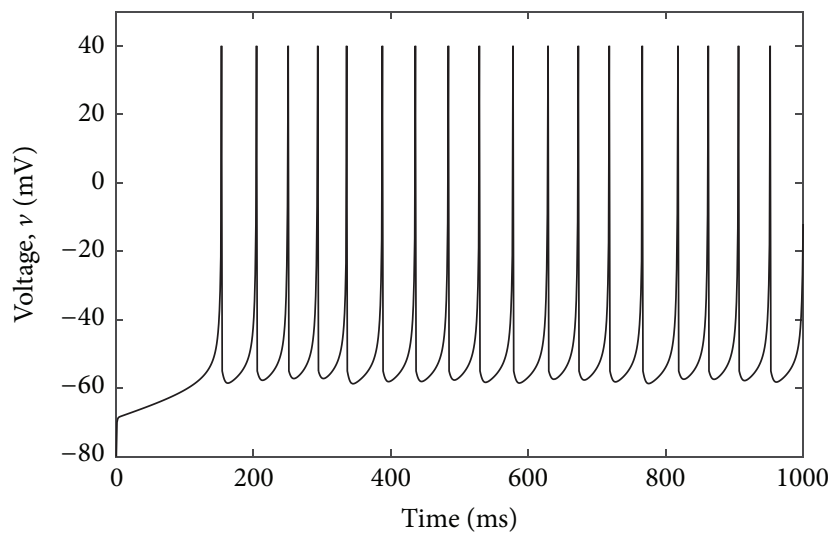

FIGURE 4: Spiking pattern of neostriatal spiny projection at $500 \mathrm{pA}$.

and 4 , respectively. To replicate the features of chattering spiking neurons it has been observed that, in [8], the resting membrane potential $v_{r}$, instantaneous threshold potential $v_{t}$, and recovery time constant $a$ are unchanged at $-60 \mathrm{mV}$, $-40 \mathrm{mV}$, and 0.03 , respectively, while membrane capacitance $C=50 \mathrm{pF}, k=1.5, b=1$, and $d=150$ are also used. The membrane potential of a typical chattering spiking neuron reaches a peak value of $v_{\text {peak }}=+25 \mathrm{mV}$ which is shown in Figure 3, as the voltage reset value $c$ is set at $-40 \mathrm{mV}$ or lower and an input current of $500 \mathrm{pA}$.

4.3. Neostriatal Spiny Projection. Neostriatal spiny projection neuron displays a prominent bistable behavior in vivo. The simulation results of the spiny neurons to current pulses have been presented in [8]. The values of $v_{t}$ and $v_{r}$ have been set at $-25 \mathrm{mV}$ and $-80 \mathrm{mV}$, respectively, while $k=1$ and $b=-20$ in order to achieve an input resistance of $30 \mathrm{M} \Omega$ and rheobase current of $300 \mathrm{pA}$. The recovery time constant $a=0.01$ which points to the slow inactivation of $\mathrm{K}^{+}$. From Figure 4, it has been observed that $v_{\text {peak }}=+40 \mathrm{mV}$ during spikes and the voltage reset value $c$ is set at $-55 \mathrm{mV}$ or lower depending on the firing frequency. A value of $d=150$ and an input current of $500 \mathrm{pA}$ ensure a reasonable match of the interspike frequencies for all the values of the sensor current.

4.4. Modified Regular Spiking. The modified regular spiking firing pattern is very much alike to that of the regular spiking but it has better dynamic range than its predecessor. The parameter values set for the extraction of the regular spiking model at $C=100 \mathrm{pF}, v_{t}=-40 \mathrm{mV}, a=0.03, b=-2$, $c=-50 \mathrm{mV}$, and $v_{\text {peak }}=+35 \mathrm{mV}$ are unchanged, while $k=0.9, v_{r}=-40 \mathrm{mV}$, and $d=150$ are changed to generate the modified regular spiking feature. Figure 5 shows firing pattern of the modified regular spiking where the input sensing current is $70 \mathrm{pA}$.

\section{Results and Discussion}

To analyze the performance of the Izhikevich neuron model, it has been designed and simulated in MATLAB simulink. The corresponding simulink model has been presented in Figure 6. The time domain signal output generated by the 


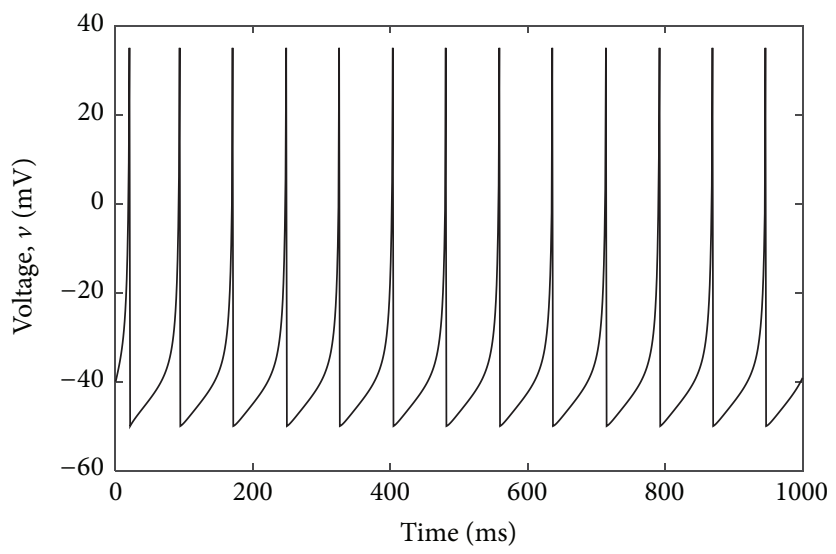

FIGURE 5: Spiking pattern of modified regular spiking at $70 \mathrm{pA}$.

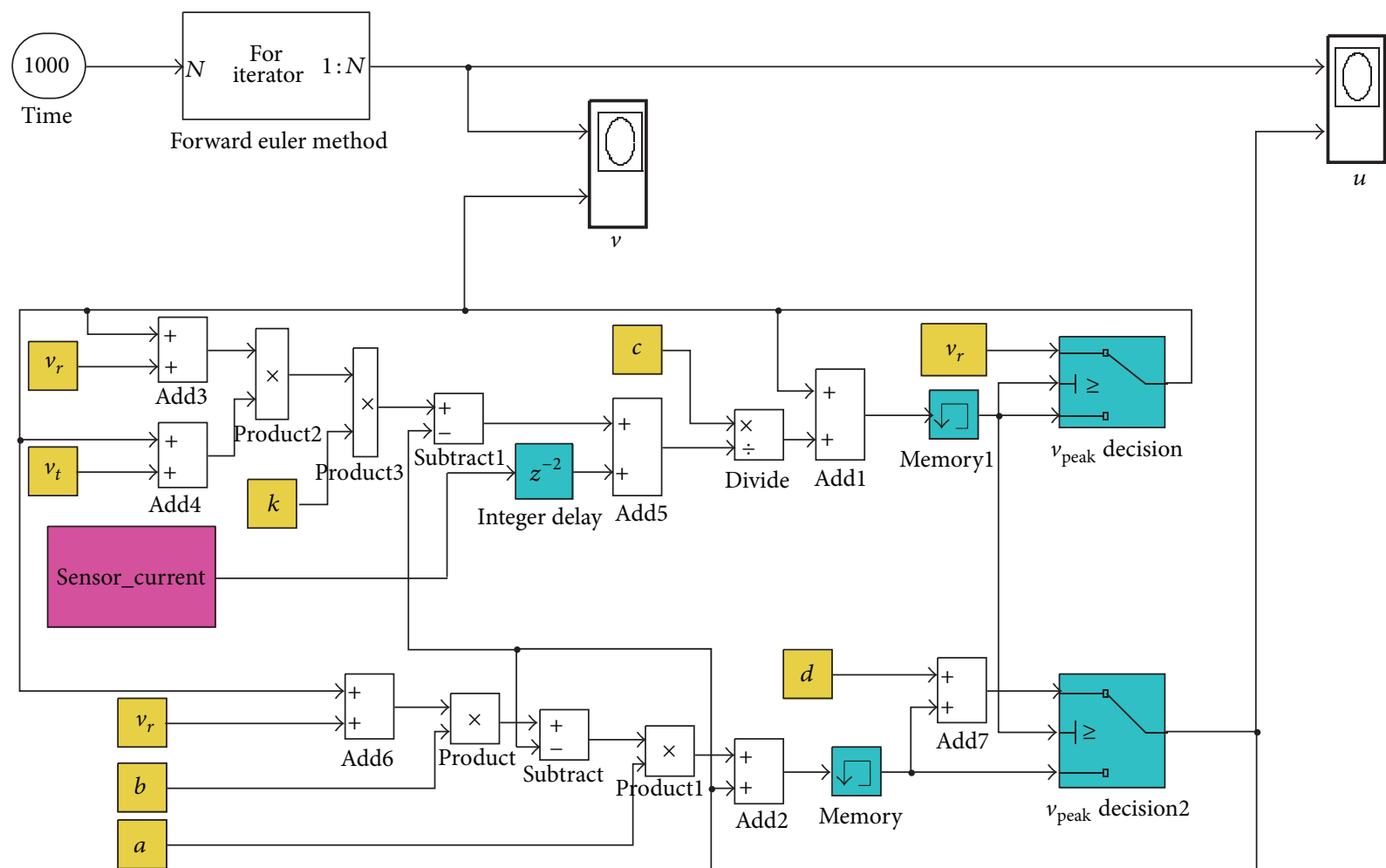

FIGURE 6: Simulink model of Izhikevich neuron model with sensing current.

neuron model has been subsequently converted to frequency domain by performing FFT.

Figure 7(a) presents the frequency spectrum of the regular spiking neuron at $70 \mathrm{pA}$ input sensor current. It can be clearly seen from the figure that the peak magnitude of the spectrum is approximately $11 \mathrm{~dB}$ which is found at $7.33 \mathrm{~Hz}$. In Figure 7(b), we find a peak magnitude of $14 \mathrm{~dB}$ at $13.33 \mathrm{~Hz}$ from the frequency spectrum of the regular spiking neuron at $100 \mathrm{pA}$ sensor current. The frequency spectrum of the regular spiking neuron at Figure 7 (c) exhibits a peak magnitude of
$15 \mathrm{~dB}$ at a frequency of $14 \mathrm{~Hz}$. The sensor current is set at $300 \mathrm{pA}$ in this instance.

Figures 8(a), 8(b), and 8(c) present the frequency spectra of the chattering neuron for sensor currents $250 \mathrm{pA}, 400 \mathrm{pA}$, and $500 \mathrm{pA}$, respectively. For a sensor current of $250 \mathrm{pA}$, the peak magnitude of $15 \mathrm{~dB}$ is observed at $14 \mathrm{~Hz}$. For increased values of sensor currents set at $400 \mathrm{pA}$ and $500 \mathrm{pA}$, the corresponding frequency spectrums show peak magnitude values of $15 \mathrm{~dB}$ and $17.3 \mathrm{~dB}$, which are found at frequencies $18 \mathrm{~Hz}$ and $24 \mathrm{~Hz}$, respectively. 
TABLE 1: The range of input sensing current and output frequency of four neuron spiking features.

\begin{tabular}{lcccc}
\hline Neuron spiking model & Range of sensing current (pA) & Input dynamic range (dB) & Output frequency (Hz) & Output linearity, $R$-square \\
\hline Regular spiking & 60 to 6000 & 40 & 5 to 1818 & 0.9816 \\
Chattering & 200 to 520 & 8.29 & 6.66 to 25 & 0.9989 \\
Neostriatal spiny projection & 400 to 6000 & 23.52 & 20 to 1818 & 0.9918 \\
Modified regular spiking & 2 to 8000 & 72.04 & 1.47 to 700 & 0.9665 \\
\hline
\end{tabular}

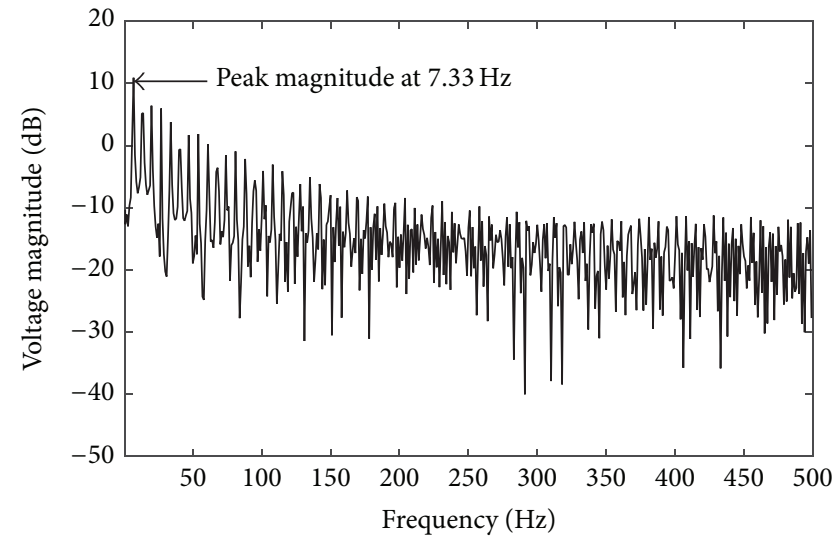

(a)

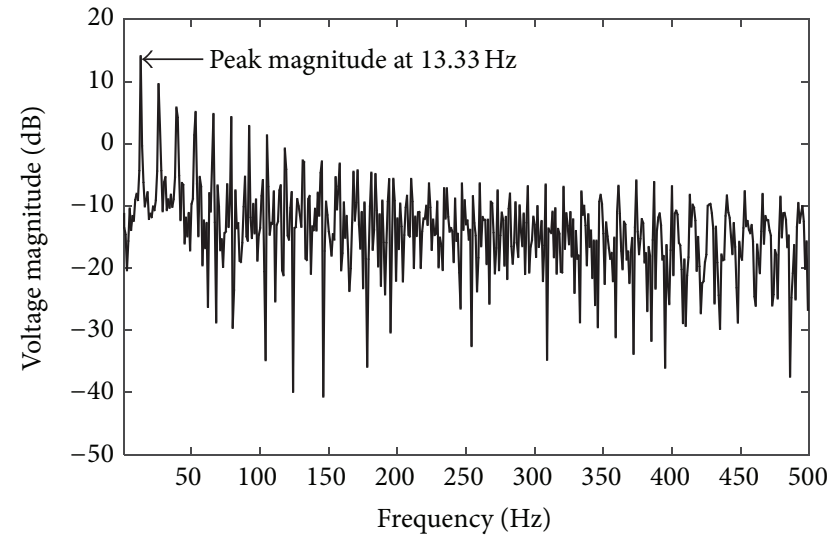

(b)

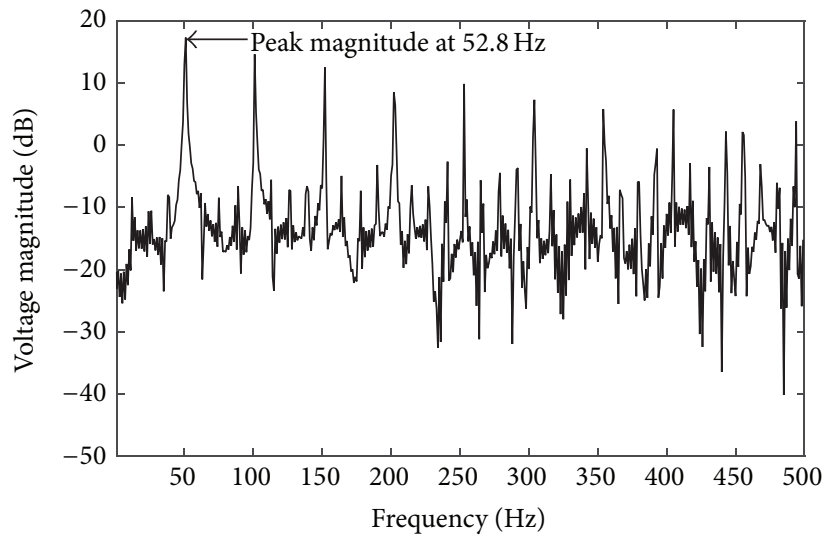

(c)

FIGURE 7: Regular spiking frequency spectrum for (a) $70 \mathrm{pA}$, (b) $100 \mathrm{pA}$, and (c) $300 \mathrm{pA}$.

The frequency spectrum of the neostriatal spiny projection is presented in Figure 9(a). For a sensor current of $500 \mathrm{pA}$ the spectrum shows a peak value of $12 \mathrm{~dB}$ at $21 \mathrm{~Hz}$. Figures 9(b) and 9(c) show the frequency spectrum of the same neuron feature where sensor currents are set at $1000 \mathrm{pA}$ and $1500 \mathrm{pA}$, respectively. It is evident from the figures that the input sensing current is increased and the frequency at which the peak magnitude is found gets shifted to a higher value of its own. Sensor currents of $1000 \mathrm{pA}$ and $1500 \mathrm{pA}$ yield peak magnitude values of $17 \mathrm{~dB}$ and $20 \mathrm{~dB}$, which are found at $62.5 \mathrm{~Hz}$ and $100 \mathrm{~Hz}$, respectively.

At last, the frequency spectrum of the modified regular spiking neuron is depicted in Figures 10(a), 10(b), and 10(c) for sensor currents $70 \mathrm{pA}, 100 \mathrm{pA}$, and $300 \mathrm{pA}$, respectively.
For a sensor current of $70 \mathrm{pA}$, the peak magnitude of $13.5 \mathrm{~dB}$ is observed at $13 \mathrm{~Hz}$. Higher values of sensor currents set at $100 \mathrm{pA}$ and $300 \mathrm{pA}$ produce peak magnitudes of $13 \mathrm{~dB}$ and $17 \mathrm{~dB}$ found at higher values of frequencies at $17 \mathrm{~Hz}$ and $40 \mathrm{~Hz}$, respectively.

Table 1 summarizes the range of input sensing currents, input sensing current dynamic ranges, output frequencies, and the output linearities for the different neuron spiking features discussed in the previous sections. Regular spiking neuron offers the best output frequency range spanning from $5 \mathrm{~Hz}$ to $1818 \mathrm{~Hz}$ while the chattering neuron has the smallest of the four, ranging from $6.66 \mathrm{~Hz}$ to $25 \mathrm{~Hz}$. On the other hand modified regular spiking model can work with the least amount of sensing current valued at only $2 \mathrm{pA}$ and ranges 


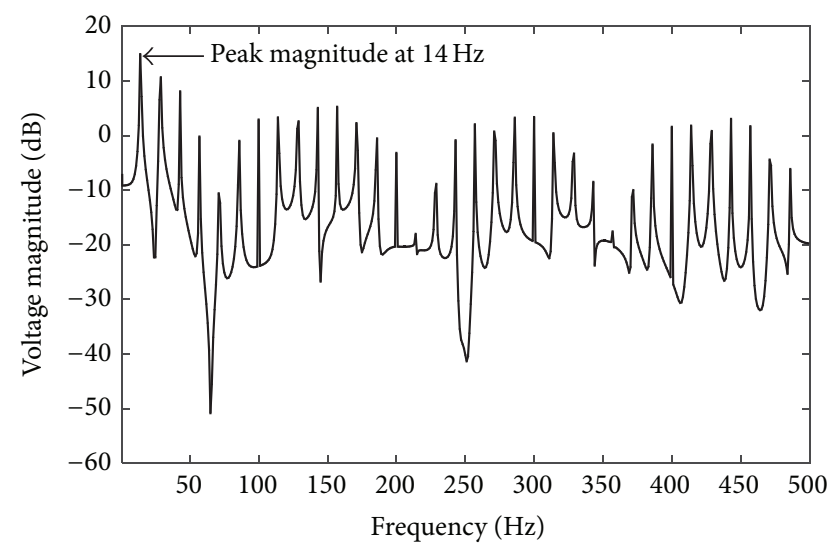

(a)

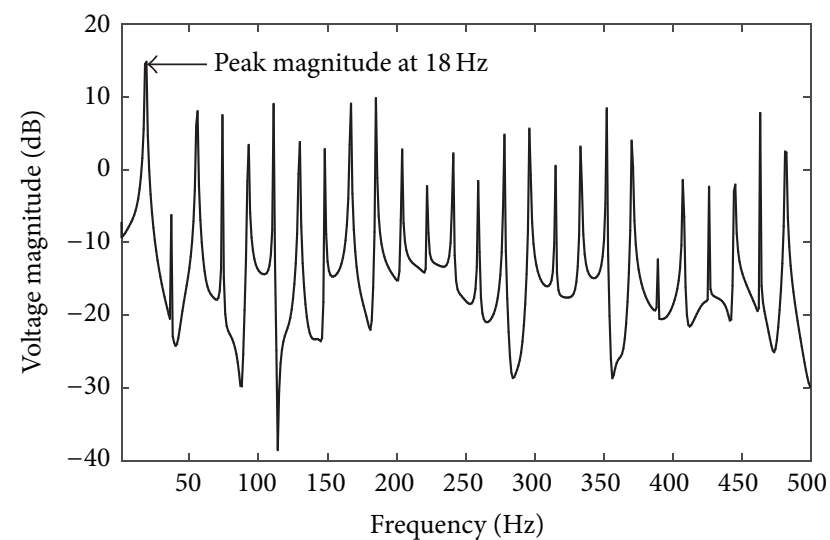

(b)

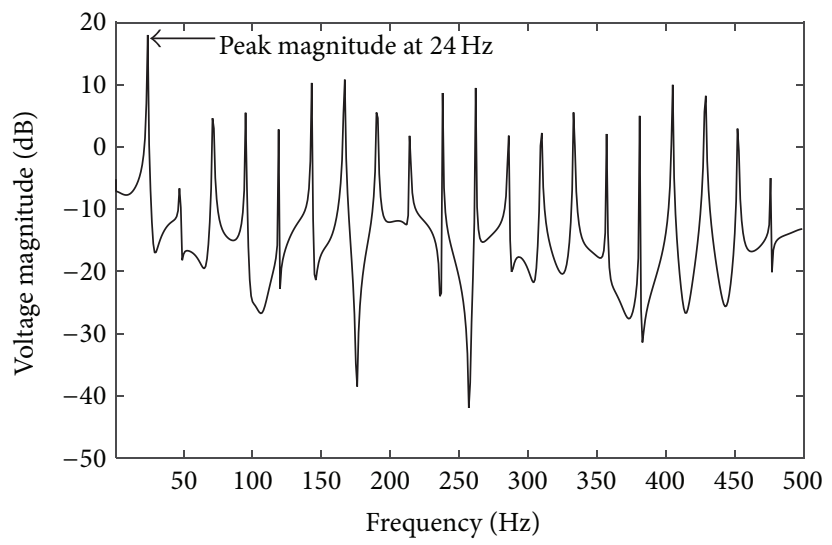

(c)

FIGURE 8: Chattering spiking frequency spectrum for (a) $250 \mathrm{pA}$, (b) $400 \mathrm{pA}$, and (c) $500 \mathrm{pA}$.

up to $8000 \mathrm{pA}$. While it has the widest input sensing current range, its output frequency range of $1.47 \mathrm{~Hz}$ to $700 \mathrm{~Hz}$ is way off from the range observed with the regular spiking neuron.

As shown in Figure 11, the input sensing current of the regular spiking varies from $60 \mathrm{pA}$ to $5000 \mathrm{pA}$, while the frequency of the output neural spikes changes from $5 \mathrm{~Hz}$ to $1818 \mathrm{~Hz}$. The $x$-axis in the figure has been set at logarithmic scale. In order to check the linearity of the input sensing current versus the output frequency, a fourth degree polynomial equation shown in (5) has been used, where $X$ is input sensing current and $Y$ is output frequency:

$$
Y=A+B 1 \times X+B 2 \times X^{2}+B 3 \times X^{3}+B 4 \times X^{4} .
$$

In (5), the fitting coefficients are set as $A=124367.68318$, $B 1=-196988.47832, B 2=114417.76648, B 3=-28983.4746$, and $B 4=2710.54107$. The result indicates that the coefficient of determination or $R$-square is 0.9816 . The value indicates a good linearity of the proposed current sensor system in response to the variation of the detected current.

Figure 12 presents the sensitivity of output frequency to the input sensing current for the chattering neuron. A third degree polynomial equation has been used to determine the linearity of this particular model, where the fitting coefficients are $A=-12200.61974, B 1=14552.11481, B 2=$ -5782.18955 , and $B 3=766.42379$, respectively. The coefficient of determination ( $R$-square) has been calculated at 0.9989 , indicating that the chattering neuron model exhibits the best linearity of the four models in this context while having the smallest ranges in both input sensing current and output frequency.

In Figure 13, the response of the neostriatal spiny projection is observed for its sensitivity of output frequency with increased amounts of input sensing currents. It shows good linearity yielding $R$-square $=0.99184$, when fourth degree polynomial equation has been employed to check its linearity. The fitting coefficients are $A=217.16676, B 1=-0.66135$, $B 2=6.31764 \times 10^{-4}, B 3=-1.90972 \times 10^{-7}$, and $B 4=$ $1.85658 \times 10^{-11}$. Lastly, Figure 14 presents similar response for the proposed modified regular spiking neuron where the input sensing current ranges from $2 \mathrm{pA}$ to $8000 \mathrm{pA}$ and the frequency of the output neural spikes fluctuates between $1.47 \mathrm{~Hz}$ and $700 \mathrm{~Hz}$. Similar to the regular spiking model, the fourth degree polynomial equation has been used to find the coefficient of determination. Fitting coefficients of 


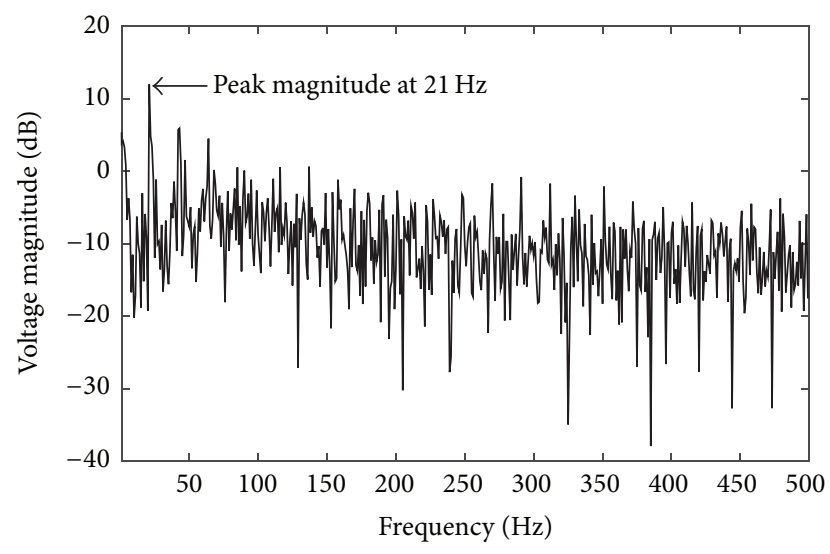

(a)

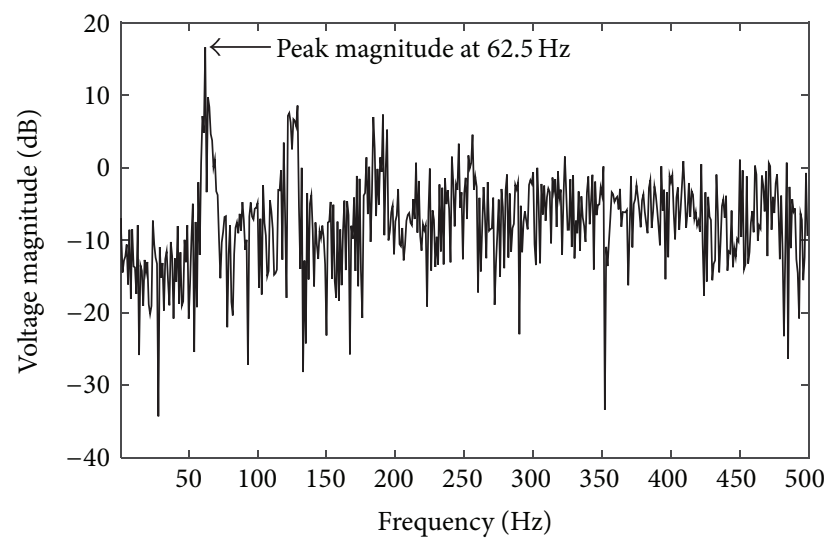

(b)

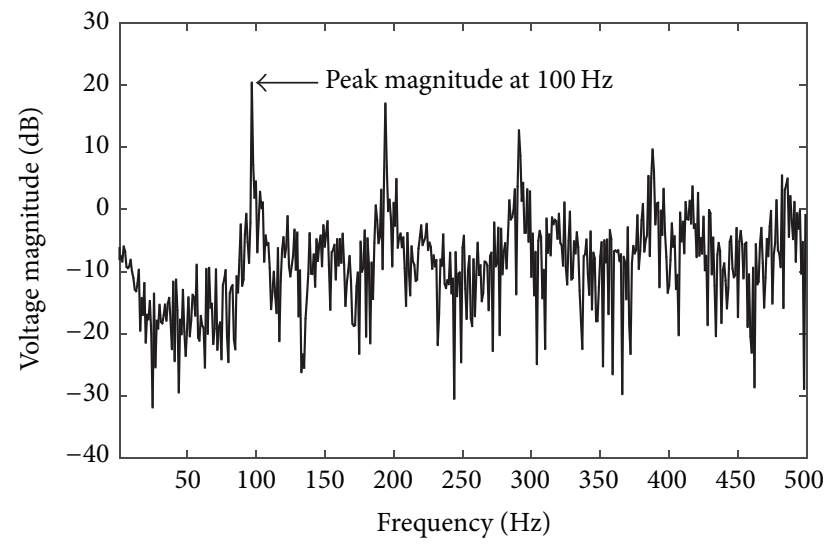

(c)

FIgURE 9: Neostriatal spiny spiking frequency spectrum for (a) $500 \mathrm{pA}$, (b) $1000 \mathrm{pA}$, and (c) $1500 \mathrm{pA}$.

$A=174.30377, B 1=-770.09961, B 2=734.05325, B 3=$ -263.0652 , and $B 4=34.31211$ have been used which generated a $R$-square value of 0.9665 .

\section{Conclusion}

In this paper an ultralow current sensor system based on a neuron model has been presented. Four neural spiking

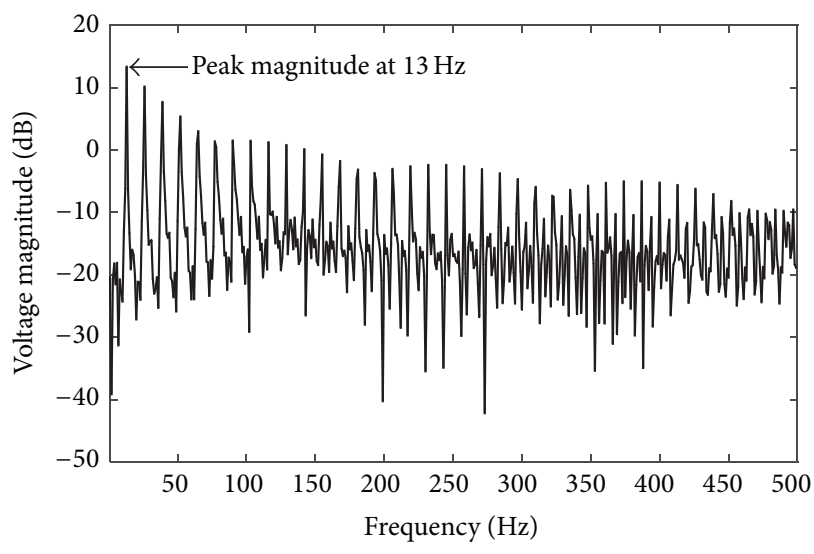

(a)

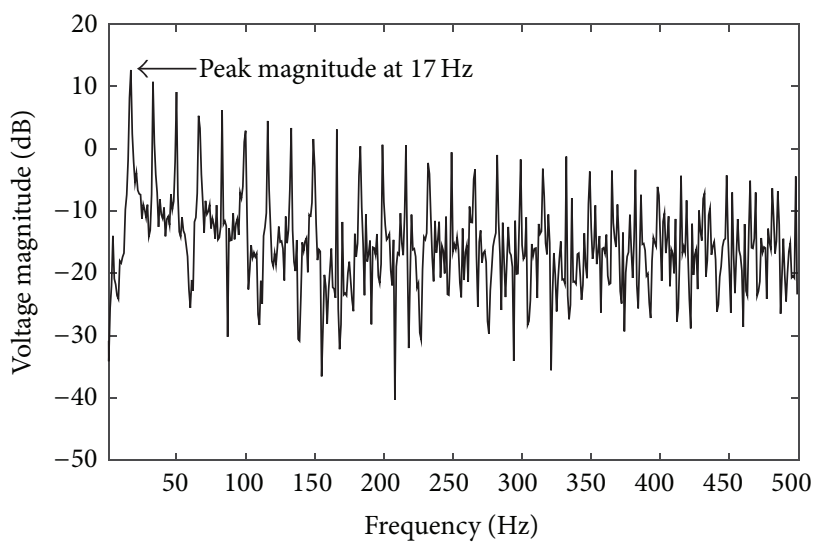

(b)

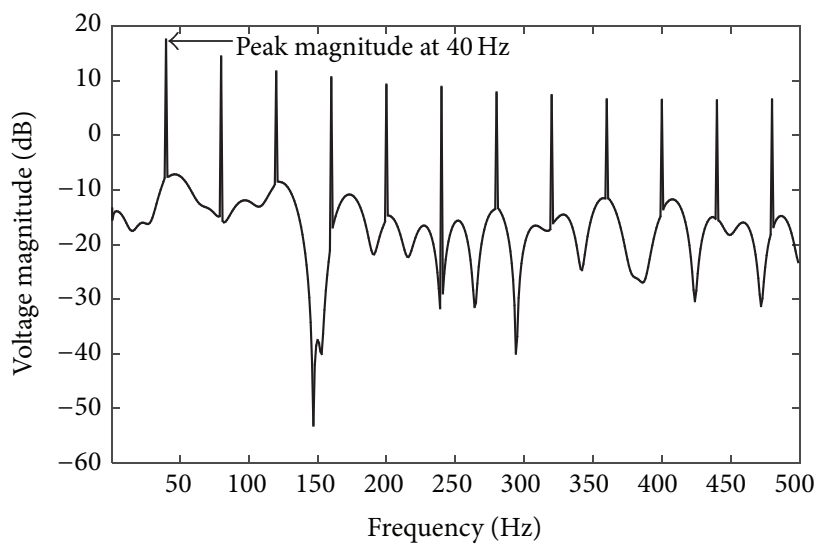

(c)

FIGURE 10: Modified regular spiking frequency spectrum for (a) $70 \mathrm{pA}$, (b) $100 \mathrm{pA}$, and (c) $300 \mathrm{pA}$.

features have been analyzed for their performances on low current detection and system linearity. The lowest value documented for the coefficient of determination is 0.9665 . The chattering neuron exhibits the best linearity with $R$ square value of 0.9989 , of the four models in this context, while having the smallest ranges in both the input sensing current and the output frequency. The proposed modified regular spiking pattern can work with the least amount of 


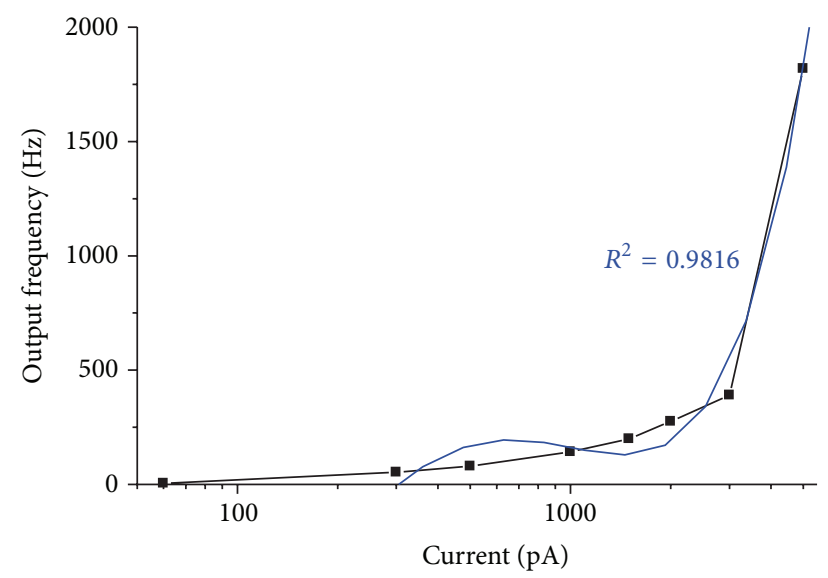

FIGURE 11: Output frequency variation of regular spiking neuron feature with input sensing current.

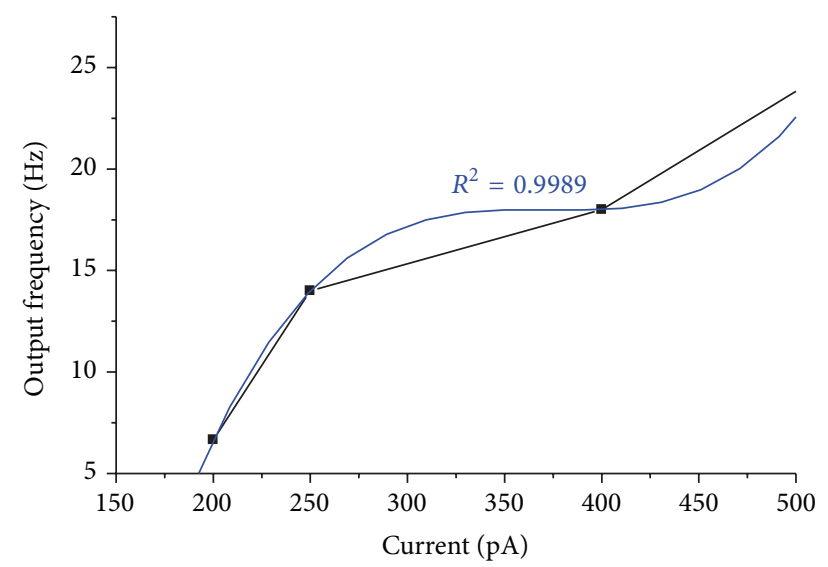

FIGURE 12: Output frequency variation of chattering neuron feature with input sensing current.

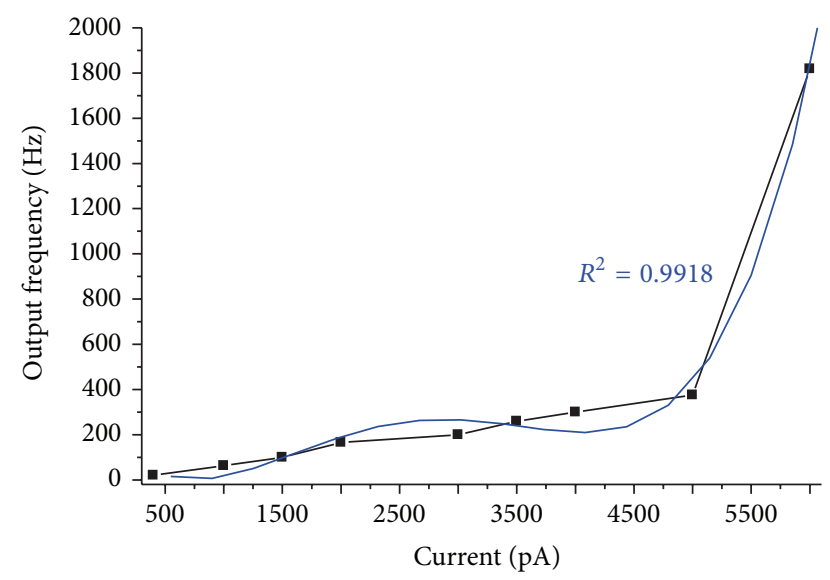

FIGURE 13: Output frequency variation of neostriatal spiny projection spiking neuron feature with input sensing current.

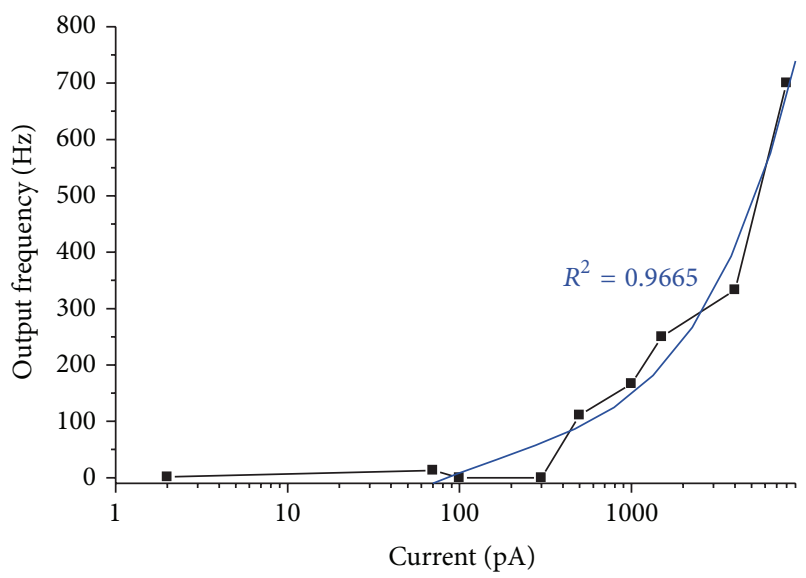

FIGURE 14: Output frequency variation of modified regular spiking neuron feature with input sensing current.

sensing current and has the widest input sensing current range of $2 \mathrm{pA}$ to $8 \mathrm{nA}$. Depending on the requirement for high linearity and enhanced dynamic range of the sensing current, the proposed sensor system can best fit for different ultralow current bioapplications.

\section{Conflict of Interests}

The authors declare that there is no conflict of interests regarding the publication of this paper.

\section{References}

[1] M. Schienle, C. Paulus, A. Frey et al., "A fully electronic DNA sensor with 128 positions and in-pixel A/D conversion," IEEE Journal of Solid-State Circuits, vol. 39, no. 12, pp. 2438-2445, 2004.

[2] S. Carrara, L. Bolomey, C. Boero et al., "Remote system for monitoring animal models with single-metabolite bio-nanosensors," IEEE Sensors Journal, vol. 13, no. 3, pp. 1018-1024, 2013.

[3] A. Mason, Y. Huang, C. Yang, and J. Zhang, "Amperometric readout and electrode array chip for bioelectrochemical sensors," in Proceedings of the IEEE International Symposium on Circuits and Systems, pp. 3562-3565, May 2007.

[4] M. Thompson, W. H. Dorn, U. J. Krull, J. S. Tauskela, E. T. Vandenberg, and H. E. Wong, "The primary events in chemical sensory perception: olfaction as a model for selective chemical sensing," Analytica Chimica Acta, vol. 180, pp. 251-269, 1986.

[5] W. W. Lee, J. Cabibihan, and N. V. Thakor, "Bio-mimetic strategies for tactile sensing," in Proceedings of the IEEE SENSORS, pp. 1-4, Baltimore, Md, USA, November 2013.

[6] Y.-G. Li and M. R. Haider, "A low-power neuromorphic CMOS sensor circuit for the implanted biomolecular detections," in Proceedings of the IEEE SENSORS, pp. 1-4, IEEE, Baltimore, Md, USA, November 2013.

[7] Q. Ma, M. R. Haider, V. L. Shrestha, and Y. Massoud, "Lowpower spike-mode silicon neuron for capacitive sensing of a biosensor," in Proceedings of the IEEE 13th Annual Wireless and Microwave Technology Conference (WAMICON '12), pp. 1-4, Cocoa Beach, Fla, USA, April 2012. 
[8] E. M. Izhikevich, Dynamical Systems in Neuroscience: The Geometry of Excitability and Bursting, The MIT Press, Cambridge, Mass, USA, 2007.

[9] M. I. Prodromidis and M. I. Karayannis, "Enzyme based amperometric biosensors for food analysis," Electroanalysis, vol. 14, no. 4, pp. 241-261, 2002.

[10] B. L. Hassler and R. M. Worden, "Versatile bioelectronic interfaces based on heterotrifunctional linking molecules," Biosensors \& Bioelectronics, vol. 21, no. 11, pp. 2146-2154, 2006.

[11] S. F. Peteu, D. Emerson, and R. M. Worden, "A Clark-type oxidase enzyme-based amperometric microbiosensor for sensing glucose, galactose, or choline," Biosensors \& Bioelectronics, vol. 11, no. 10, pp. 1059-1071, 1996.

[12] S. F. Peteu, M. T. Widman, and R. M. Worden, "In situ mapping of community-level cellular response with catalytic microbiosensors," Biosensors \& Bioelectronics, vol. 13, no. 11, pp. 1197-1203, 1998.

[13] M. Breten, T. Lehmann, and E. Bruun, "Integrating data converters for picoampere currents from electrochemical transducers," in Proceedings of the IEEE Internaitonal Symposium on Circuits and Systems, vol. 5, pp. 709-712, May 2000.

[14] T. Gregory Drummond, M. G. Hill, and J. K. Barton, "Electrochemical DNA sensors," Nature Biotechnology, vol. 21, no. 10, pp. 1192-1199, 2003.

[15] Z. Li, Y. Chen, X. Li, T. I. Kamins, K. Nauka, and R. S. Williams, "Sequence-specific label-free DNA sensors based on silicon nanowires," Nano Letters, vol. 4, no. 2, pp. 245-247, 2004.

[16] U. Schenk, M. Frascoli, M. Proietti et al., "ATP inhibits the generation and function of regulatory $\mathrm{T}$ cells through the activation of purinergic P2X receptors," Science Signaling, vol. 4, no. 162, article ral2, 11 pages, 2011.

[17] S. M. Rothman and J. W. Olney, "Glutamate and the pathophysiology of hypoxic-ischemic brain damage," Annals of Neurology, vol. 19, no. 2, pp. 105-111, 1986.

[18] X. Kang, J. Wang, H. Wu, I. A. Aksay, J. Liu, and Y. Lin, "Glucose Oxidase-graphene-chitosan modified electrode for direct electrochemistry and glucose sensing," Biosensors \& Bioelectronics, vol. 25, no. 4, pp. 901-905, 2009.

[19] V. Erokhin, S. Carrara, H. Amenitch, S. Bernstorff, and C. Nicolini, "Semiconductor nanoparticles for quantum devices," Nanotechnology, vol. 9, no. 3, pp. 158-162, 1998.

[20] S. Paddeu, M. K. Ram, S. Carrara, and C. Nicolini, "LangmuirSchaefer films of a poly(o-anisidine) conducting polymer for sensors and displays," Nanotechnology, vol. 9, no. 3, pp. 228-236, 1998.

[21] A. Guiseppi-Elie, C. Lei, and R. H. Baughman, "Direct electron transfer of glucose oxidase on carbon nanotubes," Nanotechnology, vol. 13, no. 5, pp. 559-564, 2002.

[22] S. Carrara, V. V. Shumyantseva, A. I. Archakov, and B. Samorì, "Screen-printed electrodes based on carbon nanotubes and cytochrome P450scc for highly sensitive cholesterol biosensors," Biosensors \& Bioelectronics, vol. 24, no. 1, pp. 148-150, 2008.

[23] S. Carrara, A. Cavallini, V. Erokhin, and G. De Micheli, "Multi-panel drugs detection in human serum for personalized therapy," Biosensors \& Bioelectronics, vol. 26, no. 9, pp. 39143919, 2011.

[24] E. M. Southern, "An improved method for transferring nucleotides from electrophoresis strips to thin layers of ionexchange cellulose," Analytical Biochemistry, vol. 62, no. 1, pp. 317-318, 1974.
[25] D. Meldrum, "Automation for genomics, sequencers, microarrays, and future trends," Genome Research, vol. 10, pp. 1288$1303,2000$.

[26] T. Vo-Dinh and B. Cullum, "Biosensors and biochips: advances in biological and medical diagnostics," Fresenius' Journal of Analytical Chemistry, vol. 366, no. 6-7, pp. 540-551, 2000.

[27] P. Hegde, R. Qi, K. Abernathy et al., "A concise guide to cDNA microarray analysis," BioTechniques, vol. 29, no. 3, pp. 548-556, 2000.

[28] D. Zhang, M. J. Anderson, M. C. Huarng, and E. C. Alocilja, "Nanoparticle-based biobarcoded DNA sensor for the rapid detection of pagA gene of Bacillus anthracis," IEEE Transactions on Nanotechnology, vol. 10, no. 6, pp. 1433-1438, 2011.

[29] E. M. Izhikevich, "Which model to use for cortical spiking neurons?" IEEE Transactions on Neural Networks, vol. 15, no. 5, pp. 1063-1070, 2004.

[30] Q. Ma, M. R. Haider, V. L. Shrestha, and Y. Massoud, "Bursting Hodgkin-Huxley model-based ultra-low-power neuromimetic silicon neuron," Analog Integrated Circuits and Signal Processing, vol. 73, no. 1, pp. 329-337, 2012. 


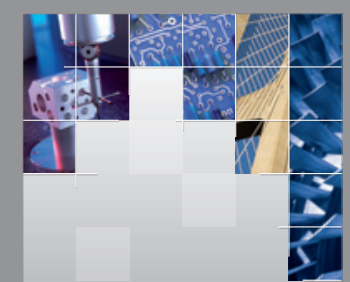

\section{Enfincering}
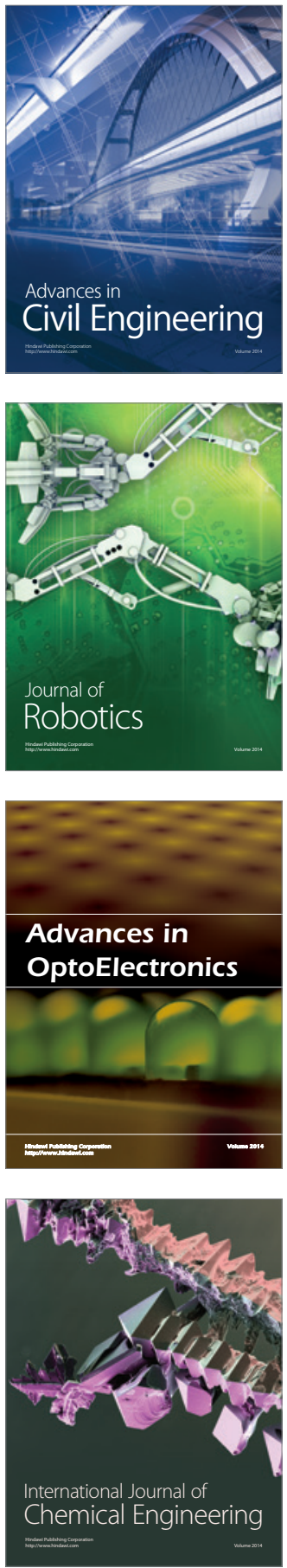

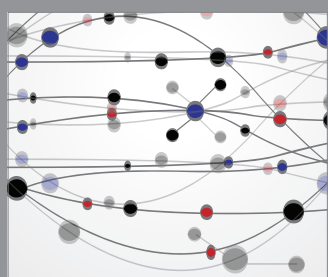

The Scientific World Journal

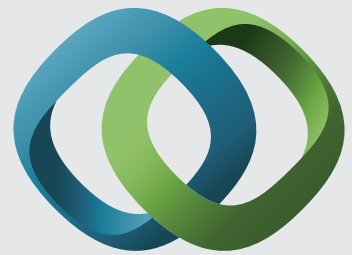

\section{Hindawi}

Submit your manuscripts at

http://www.hindawi.com
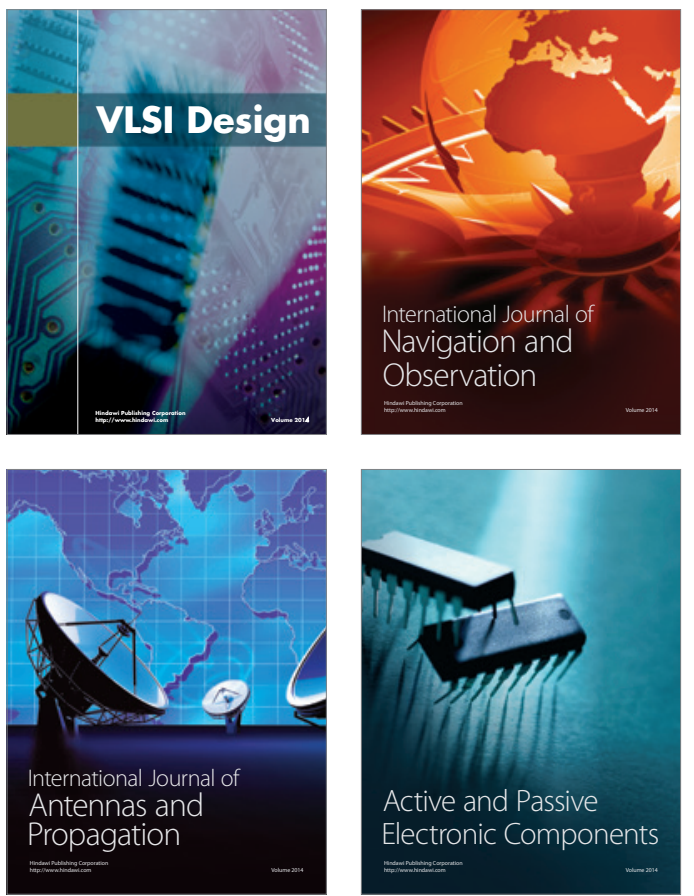
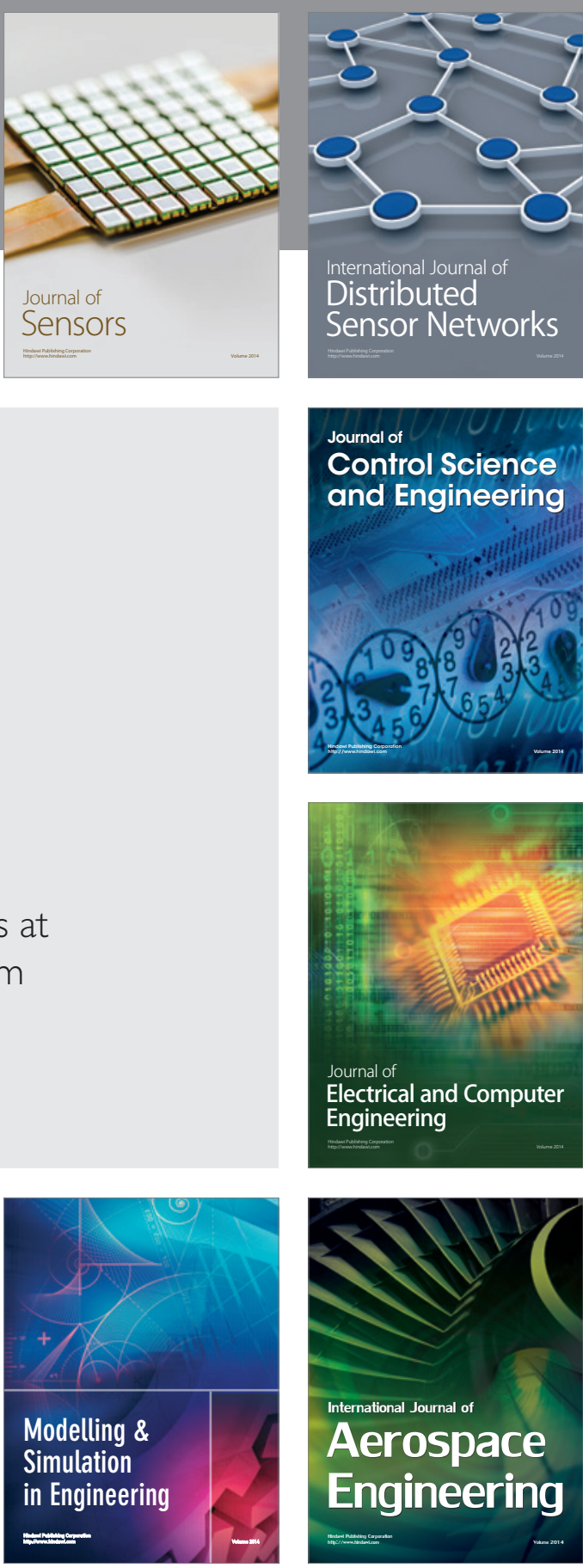

International Journal of

Distributed

Sensor Networks

Journal of

Control Science

and Engineering
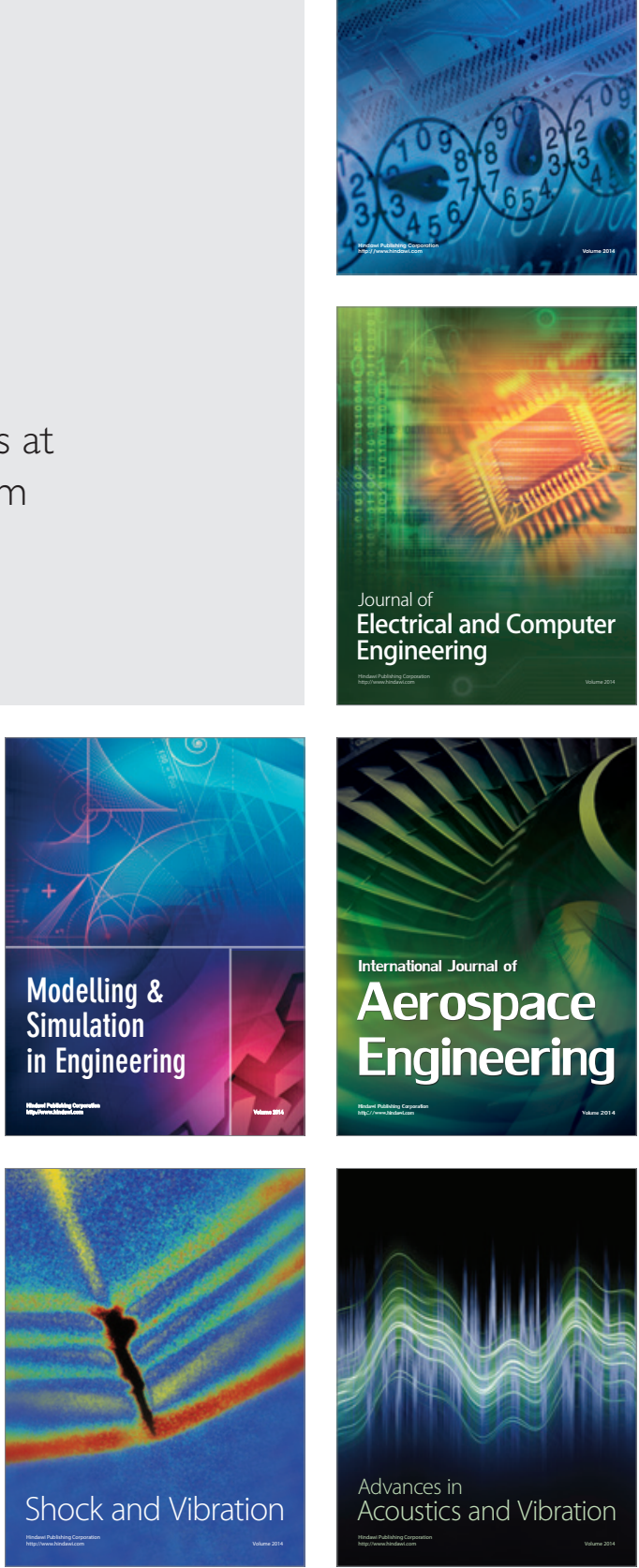